\title{
ON THE LOWER TAIL VARIATIONAL PROBLEM FOR RANDOM GRAPHS
}

\author{
YUFEI ZHAO
}

\begin{abstract}
We study the lower tail large deviation problem for subgraph counts in a random graph. Let $X_{H}$ denote the number of copies of $H$ in an Erdös-Rényi random graph $\mathcal{G}(n, p)$. We are interested in estimating the lower tail probability $\mathbb{P}\left(X_{H} \leq(1-\delta) \mathbb{E} X_{H}\right)$ for fixed $0<\delta<1$.

Thanks to the results of Chatterjee, Dembo, and Varadhan, this large deviation problem has been reduced to a natural variational problem over graphons, at least for $p \geq n^{-\alpha_{H}}$ (and conjecturally for a larger range of $p$ ). We study this variational problem and provide a partial characterization of the so-called "replica symmetric" phase. Informally, our main result says that for every $H$, and $0<\delta<\delta_{H}$ for some $\delta_{H}>0$, as $p \rightarrow 0$ slowly, the main contribution to the lower tail probability comes from Erdős-Rényi random graphs with a uniformly tilted edge density. On the other hand, this is false for non-bipartite $H$ and $\delta$ close to 1 .
\end{abstract}

\section{BACKGROUND}

We consider large deviations of subgraph counts in Erdős-Rényi random graphs. Fix a graph $H$, and let $X_{H}$ denote the number of copies of $H$ in an Erdös-Rényi random graph $\mathcal{G}(n, p)$. For a fixed $\delta>0$, the problem is to estimate the probabilities

$$
\begin{array}{ll}
\text { (upper tail) } & \mathbb{P}\left(X_{H} \geq(1+\delta) \mathbb{E} X_{H}\right) \quad \text { and } \\
\text { (lower tail) } & \mathbb{P}\left(X_{H} \leq(1-\delta) \mathbb{E} X_{H}\right) .
\end{array}
$$

This problem has a long history (see $[6]$ and its references). For the order of the logarithm of the tail probability, the upper tail problem is considered more difficult and it was resolved only fairly recently 6, 12. We are now interested in the finer question of determining the large deviation rate, or equivalently the first order asymptotics of the logarithm of the tail probability.

Chatterjee and Varadhan [10] (the dense setting, with $p$ constant) and more recently Chatterjee and Dembo [7] (the sparse setting, with $p \rightarrow 0$ and $p \geq n^{-\alpha_{H}}$ for some $\alpha_{H}>0$ ) showed that this large deviation problem reduces to a natural variational problems in the space of graphons, which are a certain type of graph limits. We begin by reviewing this connection, and then we shift our attention to analyzing the variational problem.

The language of graph limits is used throughout our discussion, so let us review some terminologies. We refer the readers to the beautifully written monograph by Lovász [24] or the original sources, e.g., 4, 5, 25, 26], for more on the subject. A graphon is a symmetric measurable function $W:[0,1]^{2} \rightarrow[0,1]$ (here symmetric means $W(x, y)=W(y, x)$ ). We write $V(H)$ and $E(H)$ to mean the vertex and edge set of a graph $H$, respectively, and $v(H)=|V(H)|$ and $e(H)=|E(H)|$ to denote their cardinalities. For any graphs $H$ and $G$, we write $\operatorname{hom}(H, G)$ to denote the number of graph homomorphisms from $H$ to $G$. We denote by $t(H, G):=\operatorname{hom}(H, G) / v(G)^{v(H)}$ the $H$-density in $G$. The $H$-density of a graphon $W$ is defined by (here $W$ could be $\mathbb{R}$-valued):

$$
t(H, W):=\int_{[0,1]^{V(H)}} \prod_{i j \in E(H)} W\left(x_{i}, x_{j}\right) \prod_{i \in V(H)} d x_{i} .
$$

The author is supported by a Microsoft Research PhD Fellowship. 
As usual, $K_{t}$ denotes the complete graph on $t$ vertices. As an example, we have

$$
t\left(K_{3}, W\right)=\int_{[0,1]^{3}} W(x, y) W(x, z) W(y, z) d x d y d z .
$$

The notion of cut distance is mentioned a few times in this paper, but it is not used in a substantial way, so we refer the readers to [24, Chapter 8] for details.

We write

$$
I_{p}(x):=x \log \frac{x}{p}+(1-x) \log \frac{1-x}{1-p}
$$

for the relative entropy function. For any function $f$, we write $\mathbb{E}[f(W)]:=\int_{[0,1]^{2}} f(W(x, y)) d x d y$.

We begin with a review of what is known for upper tails. In the dense case, for fixed $0<p \leq$ $q<1$, it was shown in 10 that as $n \rightarrow \infty$,

$$
\log \mathbb{P}\left(t(H, \mathcal{G}(n, p)) \geq q^{e(H)}\right)=-(1+o(1)) \frac{n^{2}}{2} \mathrm{UT}_{p}(H, q),
$$

where $\mathrm{UT}_{p}(H, q)$, for any graph $H$, is given by the upper tail variational problem:

$$
\mathrm{UT}_{p}(H, q):=\left\{\begin{array}{l}
\text { minimize } \mathbb{E}\left[I_{p}(W)\right] \\
\text { subject to } t(H, W) \geq q^{e(H)} .
\end{array}\right.
$$

Here $W$ is taken over all graphons. We shall use $\mathrm{UT}_{p}(H, q)$ to refer to the variational problem as well as its value, i.e., $\min \left\{\mathbb{E}\left[I_{p}(W)\right]: t(H, W) \geq q^{e(H)}\right\}$ (it is known that the minimum is always attained by some $W$; see Lemma 5.1 below).

Furthermore, as shown in [10, Theorem 3.1], the set of minimizing $W$ in $\mathrm{UT}_{p}(H, q)$ represents the most likely models for $\mathcal{G}(n, p)$ conditioned on the rare event of $t(H, \mathcal{G}(n, p)) \geq q^{e(H)}$, in the sense that the random graph conditioned on this rare event will be exponentially more likely to be close to the minimizing set of $W$ 's in terms of cut distance. This motiviates the study of $\mathrm{UT}_{p}(H, q)$ and related variational problems.

We currently have few tools for solving variational problems of the type $(1.2)$. Note that $W \equiv q$ alway satisfies the constraint in (1.2). We focus on the basic question: does the constant graphon $W \equiv q$ minimize $\mathrm{UT}_{p}(H, q)$ ? The answer depends on the graph $H$ and parameters $(p, q)$. For a fixed $H$, we wish to determine for each $(p, q)$ whether $\mathrm{UT}_{p}(H, q)=I_{p}(q)$ or $\mathrm{UT}_{p}(H, q)<I_{p}(q)$, and in the former case, whether the constant function $W \equiv q$ is the unique minimizer 1 . The separation of these two cases can be illustrated via a phase diagram, as in Figure 1, by plotting the phases in the $(p, q)$-plane according to the behavior of $\mathrm{UT}_{p}(H, q)$.

The constant graphon $W \equiv q$ is the limit of random graphs $\mathcal{G}(n, q)$ as $n \rightarrow \infty$, so if it were the unique minimizer of $\mathrm{UT}_{p}(H, q)$ then $\mathcal{G}(n, p)$, conditioned on having $H$-density at least $q^{e(H)}$, approaches the typical $\mathcal{G}(n, q)$ in cut distance; this is not the case when $W \equiv q$ is not a minimizer. Borrowing language from statistical physics, informally, when $W \equiv q$ is a minimizer we say that there is replica symmetry ${ }^{2}$, and otherwise there is symmetry breaking.

In a previous paper with Lubetzky [28], we completely identified the upper tail replica symmetric phase whenever $H$ is a $d$-regular graph. The phase diagram depends only on $d$. The diagram for

\footnotetext{
${ }^{1}$ We identify graphons differing on a measure zero set, as well as up to a measure-preserving transformation on $[0,1]$, i.e., $W$ is identified with $W^{\sigma}(x, y):=W(\sigma(x), \sigma(y))$ where $\sigma:[0,1] \rightarrow[0,1]$ is measure-preserving.

${ }^{2}$ There is a subtle issue of uniqueness of the minimizer. When the constant graphon $W \equiv q$ is the unique minimizer, $\mathcal{G}(n, q)$ represents the most likely model for the conditioned random graph (in terms of cut metric). However, it may be the case that $W \equiv q$ is a non-unique minimizer (which provably does not happen for $\mathrm{UT}_{p}\left(K_{3}, q\right.$ ) but I suspect that it does happen for the corresponding lower tail problem $\mathrm{LT}_{p}\left(K_{3}, q\right)$ ). When there are multiple distinct minimizers to the variational problem, all minimizers give rise to the same exponential rate, but one minimizer might still dominate by a lower order $\exp \left(o\left(n^{2}\right)\right)$ factor, which I do not know how to discern purely from the variational problem.
} 


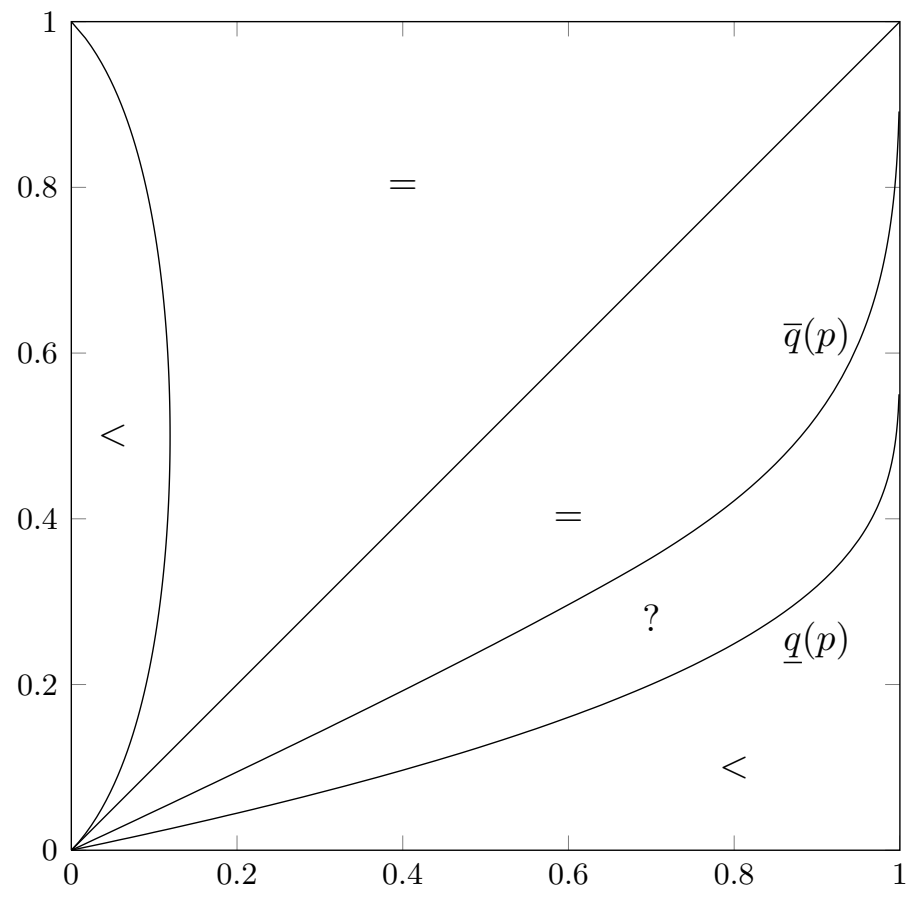

FiguRE 1. The phase diagram for triangle density upper tail variational problem $\mathrm{UT}_{p}\left(K_{3}, q\right)$ (when $q>p$ ) and lower tail variational problem $\mathrm{LT}_{p}\left(K_{3}, q\right)$ (when $q<$ $p)$. In regions marked "=", the constant graphon $W \equiv q$ is the unique minimizer to the variational problem. In regions marked " $<$ ", the constant graphon does not minimize the variational problem. The region marked "?" is unresolved. The boundary curves $\bar{q}$ and $\underline{q}$ are from Theorem 2.1 .

$H=K_{3}$ is shown in Figure 1 in the upper portion (i.e., $q>p$ ) of the diagram ${ }^{3}$, The lower portion of the diagram illustrates new results in paper concerning the lower tail problem.

In this paper we study the corresponding lower tail variational problem. For $0 \leq q \leq p \leq 1$, let

$$
\operatorname{LT}_{p}(H, q):=\left\{\begin{array}{l}
\text { minimize } \mathbb{E}\left[I_{p}(W)\right] \\
\text { subject to } t(H, W) \leq q^{e(H)} .
\end{array}\right.
$$

The connections between the large deviation problem and the variational problem discussed earlier hold for the lower tail just as they do for the upper tail. For example, as in (1.1), for fixed $0 \leq q \leq p \leq 1$, we have

$$
\log \mathbb{P}\left(t(H, \mathcal{G}(n, p)) \leq q^{e(H)}\right)=-(1+o(1)) \frac{n^{2}}{2} \mathbf{L T}_{p}(H, q) .
$$

As observed in [28], if $H$ is a bipartite graph satisfying Sidorenko's conjecture [36], which asserts that $t(H, W) \geq \mathbb{E}[W]^{e(H)}$ for all graphons $W$, then from the constraint of $\mathbf{L T}_{p}(H, q)$ we deduce $\mathbb{E}[W] \leq q$. Since $I_{p}(\cdot)$ is a convex function, from $\mathbb{E}[W] \leq q$ it follows that $W \equiv q$ is the unique minimizer of $\mathrm{LT}_{p}(H, q)$. Sidorenko's conjecture remains open though it has been proved for certain families of bipartite graphs $H$ such as trees, cycles, hypercubes, and bipartite graphs containing one

\footnotetext{
${ }^{3}$ The boundary curve for the upper tail phase diagram for $K_{3}$ is given by the equation $\left(1+\left(q^{-1}-1\right)^{1 /(1-2 q)}\right) p=1$.

${ }^{4}$ The first unsettled case of Sidorenko's conjecture is for the graph $H$ being $K_{5,5}$ with a Hamiltonian cycle removed (this $H$ is sometimes called a "Möbius strip"). There is some sentiment in the community that Sidorenko's conjecture may be false for this graph.
} 
vertex adjacent to all vertices on the opposite side [11,14,21,23,37]. Even if Sidorenko's conjecture were false, it could still be true that $W \equiv q$ minimizes $\operatorname{LT}_{p}(H, q)$ for every bipartite $H$.

For the first non-bipartite case, namely $K_{3}$, new results in this paper partially characterize the lower tail phase diagram, as depicted in Figure 1. The region marked "?" remains unresolved. For other non-bipartite graph $H$, it is possible to draw similar partially identified phase diagrams using techniques in this paper. We will pay special attention to the slopes of the boundary curves at the origin.

The lower tail variational problem seems to be harder than the corresponding upper tail problem ${ }^{5}$ By analogy, for the classical extremal graph theory problem of determining the range of possible triangle densities in a graph of fixed edge density, the maximization problem (analogous to upper tail) follows as a corollary of the classic Kruskal-Katona theorem [18,22]6 , whereas the corresponding minimization problem (analogous to lower tail) was solved only relatively recently by Razborov 34 using his flag algebra machinery (also later solved for $K_{4}$ by Nikiforov [29] and all $K_{t}$ by Reiher [35]). Furthermore, the qualitative nature of the phase transition seems to be different for the upper tail and the lower tail. It seems likely that the optimizing graphon $W$ changes continuously as $(p, q)$ crosses upper tail phase boundary, while discontinuously for the lower tail.

The sparse setting, with $p=p_{n} \rightarrow 0$ and $q / p$ kept constant, is more difficult. Using powerful new methods, Chatterjee and Dembo [7] showed that the large deviation problem in sparse random graphs also reduces to the natural variational problem 7 , provided that $p \geq n^{-\alpha_{H}}$ for some explicit $\alpha_{H}>0$. A similar conclusion can be made about the lower tail variational problem using their techniques. With Lubetzky [27] we obtained the following asymptotic solution to the corresponding variational problem: for every fixed $\delta>0$,

$$
\lim _{p \rightarrow 0} \frac{\mathrm{UT}_{p}\left(K_{3},(1+\delta)^{1 / 3} p\right)}{p^{2} \log (1 / p)}=\min \left\{\delta^{2 / 3}, \frac{2}{3} \delta\right\},
$$

and as a corollary, as long as $p=p_{n} \rightarrow 0$ with $p_{n} \geq n^{-1 / 42} \log n$, we have

$$
\mathbb{P}\left(t\left(K_{3}, \mathcal{G}(n, p)\right) \geq(1+\delta) p^{3}\right)=\exp \left(-(1-o(1)) \min \left\{\frac{\delta^{2 / 3}}{2}, \frac{\delta}{3}\right\} n^{2} p^{2} \log \frac{1}{p}\right) .
$$

In this paper, we also study the lower tail variational problem as $p \rightarrow 0$. A nice feature of the lower tail problem in the sparse limit is that instead of being concerned with the entire phase boundary curve, we can focus on its slope at the origin 8

The lower tail problem was also recently analyzed by Janson and Warnke [17] from a completely different perspective (not relating to the variational problem). In the triangle case, for $n^{-1 / 2} \ll$ $p \rightarrow 0$, they were able to determine the large deviation rate of $\mathbb{P}\left(t\left(K_{3}, \mathcal{G}(n, p)\right) \leq(1-\delta) p^{3}\right)$ for the two extremes $\delta=o(1)$ and $\delta=1-o(1)$. They left as an open question what happens for fixed $\delta \in(0,1)$, which is the subject of this paper.

There are other variants of the variational problem being studied in literature. For exponential random graphs, see $[1,3,9,20,28,33,39,44]$. For the variational problem where several subgraph densities are simultaneously constrained (e.g., edge and triangle densities both fixed), see $2,19,30$ 32 .

\footnotetext{
${ }^{5}$ despite that the probabilistic problem of determining the order of $\log \mathbb{P}\left(t\left(K_{3}, \mathcal{G}(n, p)\right)\right)$ when $p=p(n) \rightarrow 0$ came much later 6, 12 compared to the corresponding lower tail result 15,16 .

${ }^{6}$ The proof of the triangle upper tail result in 28 actually uses some form of strengthening of the Kruskal-Katona result, as we explain in Section 3 .

${ }^{7}$ Some minor modifications needs to made to the formulation variational problem $\mathrm{LT}_{p}(H, q)$ in order to match the statements in 7], namely that we only consider grpahons that correspond to weighted graphs on $n$ vertices. This difference is minor and does not affect the rest of this paper.

${ }^{8}$ For the upper tail boundary curve, the slope at the origin is always 1.
} 
Section 2 contains statements of the results. Section 3 reviews the techniques used in proof of the upper tail results from [28]. Section 4 concerns the upper tail problem for triangle densities. Section 5 concerns general $H$-densities. The methods in Sections 4 and 5 are different since the techniques for triangles seem to be quantitatively superior but do not extend to all graphs. Section 6 concludes with some open problems.

\section{Results}

2.1. Triangle density. Here is our main result concerning the lower tail variational problem $\mathrm{LT}_{p}\left(K_{3}, q\right)$ for triangle densities. See Figure 1 .

Theorem 2.1. There exist functions $q, \bar{q}:(0,1) \rightarrow(0,1)$ satisfying $0<q(p) \leq \bar{q}(p) \leq p$ for $0<p<1$ with the following properties. Whenever $\bar{q}(p)<q<p$, the constant graphon $W \equiv q$ is the unique minimizer for $\operatorname{LT}_{p}\left(K_{3}, q\right)$. Whenever $0<q<\underline{q}(p)$, the constant graphon $W \equiv q$ does not minimize $\mathrm{LT}_{p}\left(K_{3}, q\right)$. Furthermore, $\lim _{p \rightarrow 0} \underline{q}(p) / p=0 . \overline{2} 09 \ldots$ while $\lim _{p \rightarrow 0} \bar{q}(p) / p=0.466 \ldots$

The two curves $\underline{q}(p)$ and $\bar{q}(p)$ are drawn in Figure 1 . The nature of $\mathrm{LT}_{p}\left(K_{3}, q\right)$ remains unresolved for $(p, q)$ between these two curves.

In Theorem 2.1 and elsewhere, $0.466 \ldots$ denotes the unique $0<r<1$ satisfying $\frac{3}{2} r \log r-r+1=$ 0 , and $0.209 \ldots$ is defined as the maximum value of $r<1$ such that that the function $f_{r}(x)$ in (4.8) (also see Figure 4) has a zero in the open interval $(0, r)$.

2.2. General subgraph density. We extend Theorem 2.1 to general subgraph counts. No serious effort is made here at optimizing the quantitative bounds.

Theorem 2.2. Let $H$ be a graph. There exists a function $\bar{q}:(0,1) \rightarrow(0,1)$ with $\lim _{p \rightarrow 0} \bar{q}(p) / p<1$ such that whenever $\bar{q}(p)<q \leq p$, the constant graphon $W \equiv q$ is the unique minimizer for $\mathrm{LT}_{p}(H, q)$.

Furthermore, if $H$ is not bipartite, then there exists a function $\underline{q}:(0,1) \rightarrow(0,1)$ with $\lim _{p \rightarrow 0} \underline{q}(p) / p>$ 0 such that whenever $0 \leq q<\underline{q}(p)$, the constant graphon $W \equiv \bar{q}$ does not minimize $\mathrm{LT}_{p}\left(K_{3}, \bar{q}\right)$.

The proof of the triangle case, Theorem 2.1, makes use of Goodman's inequality [13]:

$$
t\left(K_{3}, W\right)+t\left(K_{3}, 1-W\right) \geq 1 / 4 .
$$

If $H$ satisfies $t(H, W)+t(H, 1-W) \geq 2^{-e(H)+1}$ for all graphons $W$ (such a graph $H$ is sometimes called "common" in the context of Ramsey multiplicities), then the same method can be used to establish regions where $W \equiv q$ is a minimizer of $\operatorname{LT}_{p}(H, q)$ (though the actual regions will not be the same as in Figure 1 due to other technical reasons). However, $t(H, W)+t(H, 1-W) \geq 2^{-e(H)+1}$ does not hold in general. For example, Thomason [38] showed that $K_{t}$ is a counterexample for all $t \geq 4$. Consequently, the proof method of Theorem 2.1 does not seem to extend to all $H$. Theorem 2.2 for general $H$ is proved using a different method, which seems quantitatively inferior to the method for triangles.

For bipartite $H$, I conjecture that there is no phase transition:

Conjecture 2.3. Let $H$ be a bipartite graph. Then the constant function $W \equiv q$ is always the unique minimizer of $\operatorname{LT}_{p}(H, q)$.

As mentioned in the introduction, the conjecture holds for any $H$ for which Sidorenko's conjecture is true, i.e., $t(H, W) \geq \mathbb{E}[W]^{e(H)}$ for all graphons $W$. Conjecture 2.3 may be true even if Sidorenko's conjecture were not. 
2.3. Sparse limit. Since $I_{p}$ is decreasing in $[0, p]$ and increasing in $[p, 1]$, any minimizing $W$ for $\operatorname{LT}_{p}(H, q)$ satisfies $0 \leq W \leq p$ almost everywhere. Let

$$
h(x):=x \log x-x+1
$$

so that

$$
\lim _{p \rightarrow 0} p^{-1} I_{p}(p x)=h(x)
$$

uniformly for $x \in[0,1]$. It follows that for every graph $H$ and $0 \leq r \leq 1$ we have

$$
\lim _{p \rightarrow 0} p^{-1} \mathrm{LT}_{p}(H, p r)=\mathrm{LT}(H, r)
$$

where

$$
\mathbf{L T}(H, r):=\left\{\begin{array}{l}
\text { minimize } \mathbb{E}[h(W)] \\
\text { subject to } t(H, W) \leq r^{e(H)} .
\end{array}\right.
$$

It would be interesting to solve this variational problem. As before, a basic question is whether the constant function $W \equiv r$ is a minimizer. Here is the main conjecture.

Conjecture 2.4. Let $H$ be a non-bipartite graph and $0 \leq r \leq 1$. There exists a $0<r_{H}^{*}<1$ so that $W \equiv r$ minimizes $\mathrm{LT}(H, r)$ if and only if $r \geq r_{H}^{*}$. Furthermore, $W$ is the unique minimizer for $\operatorname{LT}(H, r)$ if and only if $r>r_{H}^{*}$.

The conjecture remains open for any non-bipartite graph $H$. For the bipartite case:

Conjecture 2.5. The constant graphon $W \equiv r$ is the unique minimizer for $\operatorname{LT}(H, r)$ for every bipartite graph $H$ and every $0 \leq r \leq 1$.

In proving Theorem 2.1 and Theorem 2.2, we obtain the following results in the direction of the above conjectures.

Theorem 2.6. If $0.466 \cdots<r \leq 1$, then the constant graphon $W \equiv r$ uniquely minimizes $\mathrm{LT}\left(K_{3}, r\right)$. If $0 \leq r<0.209 \ldots$, then the constant graphon $W \equiv r$ does not minimize $\mathrm{LT}\left(K_{3}, r\right)$.

I conjecture that, in Conjecture 2.4. $r_{K_{3}}^{*}=0.209 \ldots$

Theorem 2.7. Let $H$ be a graph. There exists $\bar{r}_{H}<1$ such that $W \equiv r$ uniquely minimizes $\mathrm{LT}(H, r)$ whenever $\bar{r}_{H} \leq r \leq 1$. If $H$ is nonbipartite, then there exists $\underline{r}_{H}>0$ such that $W \equiv r$ does not minimize $\operatorname{LT}(H, r)$ for $0 \leq r<\underline{r}_{H}$.

Combining these results with the framework of Chatterjee and Dembo [7], we obtain

Corollary 2.8. Let $H$ be a graph. There is some explicit $\alpha_{H}>0$ so that for $p=p_{n} \rightarrow 0$ with $p \geq n^{-\alpha}$, the following large deviation results hold.

There exists $\bar{r}_{H}<1$ so that for all $r \in\left(\bar{r}_{H}, 1\right)$,

$$
\lim _{n \rightarrow \infty} \frac{2}{n^{2} p} \log \mathbb{P}\left(t(H, \mathcal{G}(n, p)) \leq(r p)^{e(H)}\right)=-h(r) .
$$

If $H$ is non-bipartite, then there exists $\underline{r}_{H}>0$ so that for all $r \in\left(0, \underline{r}_{H}\right)$,

$$
\liminf _{n \rightarrow \infty} \frac{2}{n^{2} p} \log \mathbb{P}\left(t(H, \mathcal{G}(n, p)) \leq(r p)^{e(H)}\right)>-h(r) .
$$

For $H=K_{3}$, we may take $\bar{r}_{K_{3}}=0.466 \ldots$ and $\underline{r}_{K_{3}}=0.209 \ldots$ 


\section{REVIEW OF THE PROOF FOR TRIANGLE UPPER TAILS}

We begin with a quick review of the proof of the upper tail result from 28, as some of the ideas are used in the proof of Theorem 2.1.

The following extension of Hölder's inequality is very useful. See [28, Corollary 3.2].

Proposition 3.1. Let $H$ be a graph with maximum degree $\Delta$. For any symmetric measurable function $W:[0,1]^{2} \rightarrow \mathbb{R}$, we have $t(H, W) \leq \mathbb{E}\left[|W|^{\Delta}\right]^{e(H) / \Delta}$. In particular, $t\left(K_{3}, W\right) \leq \mathbb{E}\left[W^{2}\right]^{3 / 2}$.

The inequality can be proved via repeated applications of Hölder's inequality (when $H=K_{3}$, it takes three applications of the Cauchy-Schwarz inequality). Observe that the inequality $t\left(K_{3}, W\right) \leq$ $\mathbb{E}\left[W^{2}\right]^{3 / 2}$ strengthens a corollary of the Kruskal-Katona theorem on the maximum possible triangle density in a graph of given edge density: $t\left(K_{3}, W\right) \leq \mathbb{E}[W]^{3 / 2}$.

The following result from [28] gives the full replica symmetric phase for $\mathrm{UT}_{p}\left(K_{3}, q\right)$, the upper tail problem for triangle densities.

Theorem 3.2. Let $0<p \leq q<1$. If the point $\left(q^{2}, I_{p}(q)\right)$ lies on the convex minorant of the function $x \mapsto I_{p}(\sqrt{x})$, then $W \equiv q$ is the unique minimizer of $\mathrm{UT}_{p}\left(K_{3}, q\right)$.

The upper tail boundary curve in Figure 1 is characterized by the condition in Theorem 3.2 See [28, Lemma 3.1] for the proof of symmetry breaking, i.e., $\mathrm{UT}_{p}\left(K_{3}, q\right)<I_{p}(q)$, to the left of the boundary curve.

Proof. By the convex minorant condition, the tangent line to the function $I_{p}(\sqrt{x})$ at $x=q^{2}$ lies below the function, so that

$$
I_{p}(\sqrt{x}) \geq I_{p}(q)+\frac{I_{p}^{\prime}(q)}{2 q}\left(x-q^{2}\right), \quad \forall x \in[0,1] .
$$

Replacing $x$ by $x^{2}$, we get

$$
I_{p}(x) \geq I_{p}(q)+\frac{I_{p}^{\prime}(q)}{2 q}\left(x^{2}-q^{2}\right), \quad \forall x \in[0,1] .
$$

Note that $I_{p}^{\prime}(q)>0$ since $q>p$.

Suppose graphon $W$ satisfies $t\left(K_{3}, W\right) \geq q^{3}$. By Proposition 3.1, we have $\mathbb{E}\left[W^{2}\right] \geq t\left(K_{3}, W\right)^{3 / 2} \geq$ $q^{2}$. Thus (3.1) implies that

$$
\mathbb{E}\left[I_{p}(W)\right] \geq I_{p}(q)+\frac{I_{p}^{\prime}(q)}{2 q}\left(\mathbb{E}\left[W^{2}\right]-q^{2}\right) \geq I_{p}(q) .
$$

This shows that $W \equiv q$ is a minimizer for $\mathrm{UT}_{p}\left(K_{3}, q\right)$, and furthermore it is not too hard to check equality conditions to verify that this is the unique minimizer.

\section{TRIAngle LOWER TAILS}

In this section we prove Theorems 2.1 and 2.6 .

4.1. Replica symmetry phase. We begin with a small modification of Goodman's theorem 13 (which is usually generally stated for $U+W \equiv 1$ ).

Lemma 4.1. If $U$ and $W$ are graphon such that $U+W \geq 2 q$ for some constant $q \geq 0$, then

$$
t\left(K_{3}, W\right)+t\left(K_{3}, U\right) \geq 2 q^{3} .
$$


Proof. By decreasing $U$ and $W$ (while remaining nonnegative), we may assume that they are graphons satisfying $U+W \equiv 2 q$. Let $U=q+X$ and $W=q-X$ for some symmetric measurable function $X:[0,1]^{2} \rightarrow \mathbb{R}$. Then

$$
\begin{aligned}
t\left(K_{3}, W\right)+t\left(K_{3}, U\right) & =t\left(K_{3}, q+X\right)+t\left(K_{3}, q-X\right) \\
& =2 q^{3}+6 q t\left(K_{1,2}, X\right) \\
& \geq 2 q^{3}+6 q(\mathbb{E}[X])^{2} \geq 2 q^{3}
\end{aligned}
$$

For any $a \in \mathbb{R}$ we write $a_{+}:=\max \{a, 0\}$. In the Proposition below, $a_{+}^{2}$ means $\left(a_{+}\right)^{2}$. The inequality (4.1) below is motivated by considering the tangent line to $x \mapsto I_{p}(2 q-\sqrt{x})$ at $x=q^{2}$, as in the proof of Theorem 3.2 .

Proposition 4.2. Let $0<q \leq p<1$ be such that

$$
I_{p}(x) \geq I_{p}(q)+\frac{-I_{p}^{\prime}(q)}{2 q}\left((2 q-x)_{+}^{2}-q^{2}\right) \quad \forall x \in[0, p] .
$$

Then $W \equiv q$ is the unique minimizer of $\mathrm{LT}_{p}\left(K_{3}, q\right)$.

Proof. Suppose $W$ satisfies $t\left(K_{3}, W\right) \leq q^{3}$. Apply Lemma 4.1 to $W$ and $U:=(2 q-W)_{+}$to obtain

$$
t\left(K_{3},(2 q-W)_{+}\right) \geq 2 q^{3}-t\left(K_{3}, W\right) \geq q^{3} .
$$

Next, apply Proposition 3.1 and we obtain

$$
\mathbb{E}\left[(2 q-W)_{+}^{2}\right] \geq t\left(K_{3},(2 q-W)_{+}\right)^{2 / 3} \geq q^{2} .
$$

By (4.1) we have (note that $I_{p}^{\prime}(q) \leq 0$ as $q \leq p$ )

$$
\mathbb{E}\left[I_{p}(W)\right] \geq I_{p}(q)+\frac{-I_{p}^{\prime}(q)}{2 q}\left(\mathbb{E}\left[(2 q-W)_{+}^{2}\right]-q^{2}\right) \geq I_{p}(q) .
$$

It follows that $\operatorname{LT}_{p}\left(K_{3}, q\right)=I_{p}(q)$. To show that $W \equiv q$ is the unique minimizer, observe that in order for any other $W$ to be a minimizer, equality must occur at every step above. In particular, if (4.1) has single point of equality, namely for $x=q$, then the uniqueness of $W$ is clear. Otherwise, one can check (details omitted, but see Figure 2) that that (4.1) has at most two points of equality, with one being $x=q$, so that if $W$ has any positive mass with value being the other point of equality, then it would be impossible for $t\left(K_{3}, W\right)=q^{3}$ to hold. This shows that $W \equiv q$ is the unique minimizer.

To derive results about the phase diagram, we shall invoke various technical statements (referred to as "Facts") about the functions $I_{p}$ and $h$. Using Fact 4.3 below we obtain the $0 \leq p \leq 1 / 2$ portion of the curve $\bar{q}$ of Theorem 2.1, which is given by the implicit equation $I_{p}(q)+\frac{1}{2} q I_{p}^{\prime}(q)=0$, and shown in Figure 1. The rest of the curve in Figure 1 is produced by numerically checking (3.1). Taking the $p \rightarrow 0$ limit of the implicit equation, we see that the slope at the origin equals to $\bar{r}=0.466 \ldots$, where $\bar{r}$ satisfies $h(\bar{r})+\frac{1}{2} \bar{r} h^{\prime}(\bar{r})=0$. This completes the proof of the replica symmetric phase in Theorem 2.1

Fact 4.3. For $0<q \leq p \leq 1 / 2$, 4.1 holds for all $x \in[0, p]$ if and only if it holds at $x=p$.

Proof. Let

$$
f(x):=f_{p, q}:=I_{p}(x)-I_{p}(q)+\frac{I_{p}^{\prime}(q)}{2 q}\left((2 q-x)_{+}^{2}-q^{2}\right) .
$$

We plotted $f$ for some representative values of $(p, q)$ in Figure 2 .

Suppose $f(p) \geq 0$. We have

$$
f^{\prime}(x)=I_{p}^{\prime}(x)-\frac{I_{p}^{\prime}(q)}{q}(2 q-x)_{+}
$$



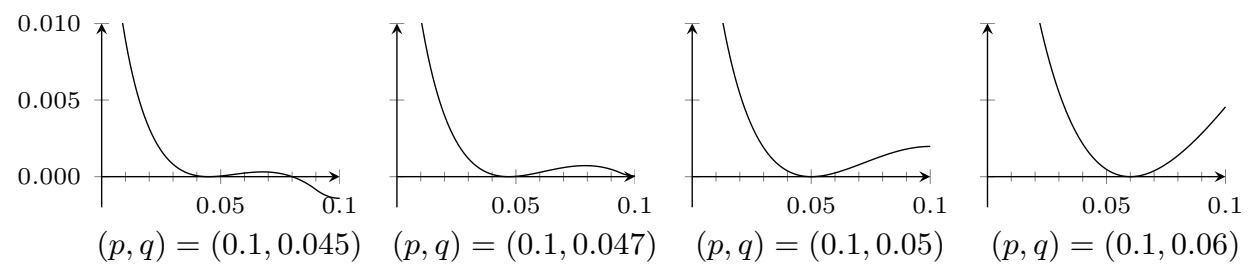

Figure 2. Plots of $f_{p, q}$ from 4.2 for $p=0.1$ and various values of $q$.

and

$$
f^{\prime \prime}(x)=I_{p}^{\prime \prime}(x)+\frac{I_{p}^{\prime}(q)}{q} 1_{x<2 q}=\frac{1}{x(1-x)}+\frac{I_{p}^{\prime}(q)}{q} 1_{x<2 q} .
$$

Since $p \leq 1 / 2, f^{\prime \prime}(x)$ is decreasing for $0<x<\min \{p, 2 q\}$. Clearly $f^{\prime \prime}$ is positive near $x=0$. We consider two cases.

Case I: $f^{\prime \prime}(x)>0$ for all $0<x<\min \{p, 2 q\}$. Then $f$ is convex on $(0, \min \{p, 2 q\})$. We know that $f(q)=f^{\prime}(q)=0$. Then $f(x) \geq 0$ for all $x \in[0, \min \{p, 2 q\}]$. If $2 q \geq p$, then we are done, otherwise, note that $f(x)=I_{p}(x)-I_{p}(q)-q I_{p}^{\prime}(q) / 2$ for $x \in[2 q, p]$, and it is decreasing on this interval. Since we assumed that $f(p) \geq 0$, we obtain $f(x) \geq 0$ for all $x \in[0, p]$.

Case II: there is some $x_{0} \in(0, \min \{p, 2 q\})$ such that $f^{\prime \prime}\left(x_{0}\right)=0$. So $f$ is convex on $\left(0, x_{0}\right)$ and concave on $\left(x_{0}, \min \{p, 2 q\}\right)$. We assumed that $f(p) \geq 0$, so $f(\min \{p, 2 q\}) \geq 0$ since if $2 q<p$ then $f$ is decreasing on $(2 q, p)$. Since $f(q)=f^{\prime}(q)=0$, an analysis of the convexity of $f$ shows that it is nonnegative on $[0, p]$.

For the sparse limit $p \rightarrow 0$, the proof of the first half of Theorem 2.6 is nearly identical. It follows from the following two propositions, whose proofs we omit.

Proposition 4.4. Let $0 \leq r \leq 1$ be such that

$$
h(x) \geq h(r)+\frac{-h^{\prime}(r)}{2 r}\left((2 r-x)_{+}^{2}-r^{2}\right) \quad \forall x \in[0,1] .
$$

Then $W \equiv r$ is the unique minimizer of $\operatorname{LT}\left(K_{3}, r\right)$.

Fact 4.5. The inequality (4.3) holds for all $x \in[0,1]$ if and only if holds for $x=1$, which holds if and only if $r \geq \bar{r}=0.466 \ldots$, where $\bar{r}$ satisfies $h(\bar{r})+\frac{1}{2} \bar{r} h^{\prime}(\bar{r})=0$.

4.2. Symmetry breaking phase. Now we explain the lower curve $q$ in Figure 1 . It is obtained by by restricting the variational problem $\mathrm{LT}_{p}\left(K_{3}, q\right)$ to graphons $W$ of the form $\mathrm{BIP}_{a, b}$, where $\mathrm{BIP}_{a, b}$, for $0 \leq a, b \leq 1$, is defined by

$$
\operatorname{BIP}_{a, b}(x, y):= \begin{cases}a & \text { if }(x, y) \in[0,1 / 2]^{2} \cup(1 / 2,1]^{2} \\ b & \text { if }(x, y) \in[0,1 / 2] \times(1 / 2,1] \cup(1 / 2,1] \times[0,1 / 2] .\end{cases}
$$

There is symmetry breaking as long as we can find $0 \leq a, b \leq p$ satisfying

$$
\mathbb{E}\left[I_{p}\left(\mathrm{BIP}_{a, b}\right)\right]=\frac{1}{2} I_{p}(a)+\frac{1}{2} I_{p}(b)<I_{p}(q)
$$

and

$$
t\left(K_{3}, \mathrm{BIP}_{a, b}\right)=\frac{1}{4} a^{3}+\frac{3}{4} a b^{2} \leq q^{3} .
$$

We can assume that $0 \leq a \leq q \leq b \leq p$, since otherwise swapping $a$ and $b$ reduces $t\left(K_{3}, W\right.$ ) (observe that $\left.t\left(K_{3}, \mathrm{BIP}_{a, b}\right)-t\left(K_{3}, \mathrm{BIP}_{b, a}\right)=\frac{1}{4}(a-b)^{3}\right)$ while keeping $\mathbb{E}\left[I_{p}(W)\right]$ constant. 

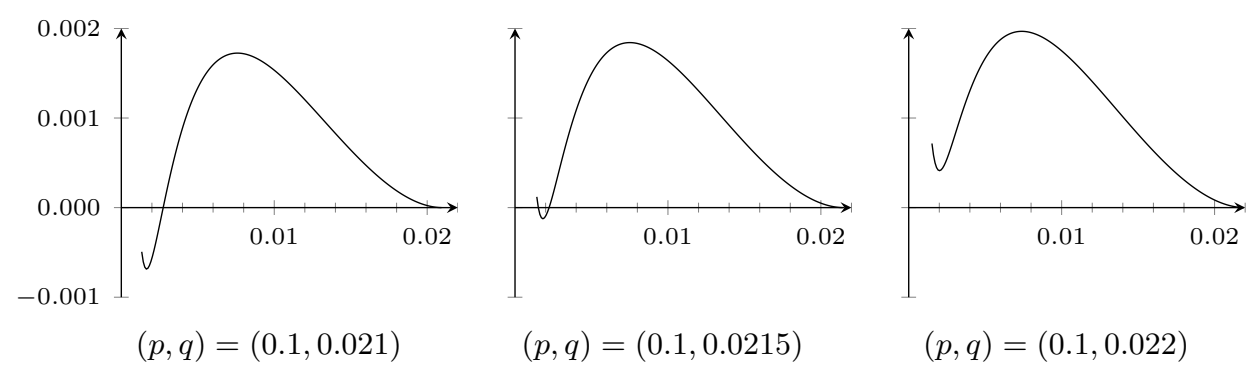

Figure 3. The plot of $f_{p, q}$ from 4.7 for $p=0.1$ and various values of $q$.
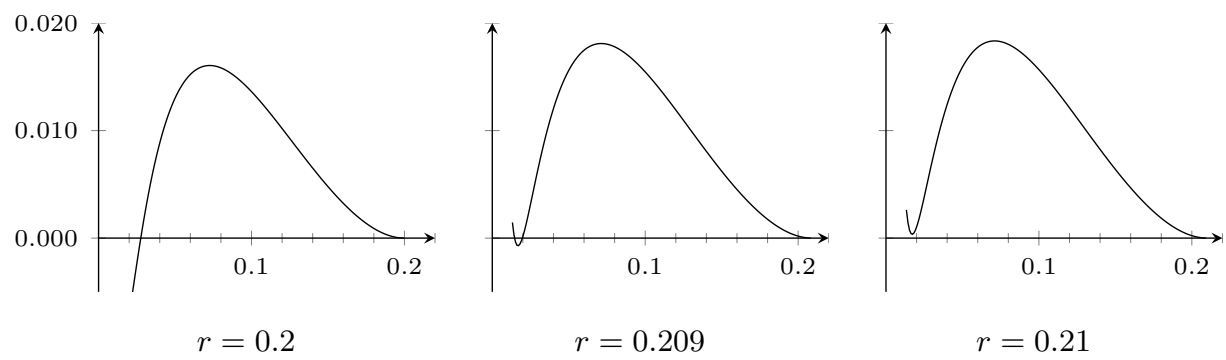

FiguRE 4. The plot of $f_{r}$ from (4.8) for various values of $r$.

Set $b=\sqrt{\left(4 q^{3}-a^{3}\right) /(3 a)}$ so that $t\left(K_{3}, \mathrm{BIP}_{a, b}\right)=q^{3}$. There is symmetry breaking if

$$
f(x):=f_{p, q}(x):=\frac{1}{2} I_{p}(x)+\frac{1}{2} I_{p}\left(\sqrt{\frac{4 q^{3}-x^{3}}{3 x}}\right)-I_{p}(q)
$$

is negative for some $0 \leq x \leq q$, where $f$ is only defined for $\sqrt{\left(4 q^{3}-x^{3}\right) /(3 x)} \leq p$. Some representative examples of $f$ are plotted in Figure 3. For every $p$, and sufficiently small $q, f(x)$ becomes negative in a region away from $x=q$.

Now we prove the claims in Theorem 2.1 more rigorously. For every $p>0$, if $q$ is sufficiently small so that $\frac{1}{2} I_{p}(0)<I_{p}(q)$, then $W=\operatorname{BIP}_{0, p}$ satisfies $t\left(K_{3}, W\right)=0$ while $\mathbb{E}\left[I_{p}(W)\right]=\frac{1}{2} I_{p}(0)<I_{p}(q)$, so that $\mathrm{LT}_{p}\left(K_{3}, q\right)<I_{p}(q)$.

The argument in the previous paragraph does not give the optimal $q$ in Theorem 2.1. To prove that $\underline{q}$ can be chosen so that $\lim _{p \rightarrow 0} \underline{q}(p) / p=0.209 \ldots$, it suffices, by (2.1), to prove the second half of Theorem 2.6, that $\operatorname{LT}\left(K_{3}, r\right)<h(r)$ for all $r<r_{1}=0.209 \ldots$ As before, we seek $0 \leq a \leq r \leq b \leq 1$ with

$$
\frac{1}{2} h(a)+\frac{1}{2} h(b)<h(r)
$$

and

$$
\frac{1}{4} a^{3}+\frac{3}{4} a b^{2} \leq r^{3}
$$

Let

$$
f(x):=f_{r}(x):=\frac{1}{2} h(x)+\frac{1}{2} h\left(\sqrt{\frac{4 r^{3}-x^{3}}{3 x}}\right)-h(r) .
$$

See Figure 4 for some examples of plots of $f_{r}$ (as before, plotted for values of $x \leq r$ satisfying $\left.\sqrt{\left(4 r^{3}-x^{3}\right) /(3 x)} \leq 1\right)$. At the critical $r=r_{1}=0.209 \ldots$, there exists $0<a_{1}<r_{1}<b_{1}<1$ such that $\frac{1}{4} a_{1}^{3}+\frac{3}{4} a_{1} b_{1}^{2}=r_{1}^{3}$ and $\frac{1}{2} h\left(a_{1}\right)+\frac{1}{2} h\left(b_{1}\right)=h\left(r_{1}\right)$. Now for any $0 \leq r<r_{1}$, let $s=r / r_{1}$, so that 
$(a, b)=\left(s a_{1}, s b_{1}\right)$ satisfies $\frac{1}{4} a^{3}+\frac{3}{4} a b^{2}=r^{3}$. Note that

$$
\begin{aligned}
h(s x) & =s x \log (s x)-s x+1 \\
& =s(x \log x-x+1)+(s \log s) x-s+1=\operatorname{sh}(x)+(s \log s) x-s+1 .
\end{aligned}
$$

We have

$$
\begin{aligned}
\frac{1}{2} h(a)+\frac{1}{2} h(b)-h(r) & =\frac{1}{2} h\left(s a_{1}\right)+\frac{1}{2} h\left(s b_{1}\right)-h\left(s r_{1}\right) \\
& =s\left(\frac{1}{2} h\left(a_{1}\right)+\frac{1}{2} h\left(b_{1}\right)-h\left(r_{1}\right)\right)+(s \log s)\left(\frac{1}{2} a_{1}+\frac{1}{2} b_{1}-r_{1}\right) \\
& <0
\end{aligned}
$$

where we know $\left(a_{1}+b_{1}\right) / 2>r$ from

$$
\begin{aligned}
\left(\frac{1}{2} a_{1}+\frac{1}{2} b_{1}\right)^{3}-r_{1}^{3} & =\left(\frac{1}{2} a_{1}+\frac{1}{2} b_{1}\right)^{3}-\frac{1}{4} a_{1}^{3}-\frac{3}{4} a_{1} b_{1}^{2} \\
& =\left(\frac{1}{2} b_{1}-\frac{1}{2} a_{1}\right)^{3}>0
\end{aligned}
$$

It follows that $\operatorname{LT}\left(K_{3}, r\right)<I_{p}(r)$ for all $0<r<r_{1}=0.209 \ldots$

\section{General subgraph lower tails}

In this section we prove Theorems 2.2 and 2.7. I will give the details only for Theorem 2.7 concerning the sparse limit $\mathrm{LT}(H, r)$ as it is somewhat cleaner and contains all the ideas. Theorem 2.2 regarding $\operatorname{LT}_{p}(H, q)$ can be proved analogously by considering sufficiently small but fixed values of $p$.

5.1. Replica symmetry. For any graph $H$ and graphon $W$, we define the functional derivative $t^{\prime}(H, W)$ to be the symmetric measurable function given by

$$
t^{\prime}(H, W)=\sum_{e \in E(H)} t_{e}(H, W)
$$

where for each $a b \in E(H)$, we define the graphon

$$
t_{a b}(H, W)\left(x_{a}, x_{b}\right):=\int_{[0,1]^{V(H) \backslash\{a, b\}}} \prod_{i j \in E(H) \backslash\{a b\}} W\left(x_{i}, x_{j}\right) \prod_{i \in V(H) \backslash\{a, b\}} d x_{i} .
$$

For example,

$$
t^{\prime}\left(K_{3}, W\right)(x, y)=3 \int_{[0,1]} W(x, z) W(y, z) d z .
$$

For any symmetric measurable $U:[0,1]^{2} \rightarrow[-1,1]$, and $\delta \rightarrow 0$, we have

$$
t(H, W+\delta U)=t(H, W)+\delta \mathbb{E}\left[t^{\prime}(H, W) U\right]+O\left(\delta^{2}\right),
$$

which justifies calling $t^{\prime}(H, W)$ the functional derivative.

Lemma 5.1. Let $H$ be a graph and $0<r<1$. The variational problem $\mathrm{LT}(H, r)$ attains its minimum for some graphon $W$, and any such $W$ satisfies the following Lagrange multiplier condition: for some $\lambda \geq 0$, one has

$$
h^{\prime}(W(x, y))+\lambda t^{\prime}(H, W)(x, y)=0, \quad \text { a.e. }-(x, y) \in[0,1]^{2} .
$$


Proof. That the minimum of $\mathrm{LT}(H, r)$ is always attained follows from the compactness of the space of graphons with respect to the cut distance and the convexity of $h$, as was already observed in 10$]^{9}$

Suppose $W$ minimizes $\mathbf{L T}(H, r)$. We claim that for any symmetric measurable function $U:[0,1]^{2} \rightarrow$ $[-1,1]$ such that $0 \leq W+U \leq 1$ and $\mathbb{E}\left[t^{\prime}(H, W) U\right]<0$, one has $\mathbb{E}\left[h^{\prime}(W) U\right] \geq 0$. Indeed, consider the graphon $W+\delta U$ for $\delta \searrow 0$. One has

$$
t(H, W+\delta U)-t(H, W)=\delta \mathbb{E}\left[t^{\prime}(H, W) U\right]+O\left(\delta^{2}\right) .
$$

Thus $t(H, W+\delta U)<t(H, W) \leq r^{e(H)}$ for sufficiently small $\delta>0$, and hence $\mathbb{E}[h(W+\delta U)] \geq$ $\mathbb{E}[h(W)]$ since $W$ minimizes $\mathrm{LT}(H, r)$. On the other hand,

$$
\lim _{\delta \rightarrow 0} \frac{\mathbb{E}[h(W+\delta U)-h(W)]}{\delta}=\mathbb{E}\left[h^{\prime}(W) U\right],
$$

so that $\mathbb{E}\left[h^{\prime}(W) U\right] \geq 0$ as claimed. The interchange of limit and expectation above can be justified by writing $U=U_{+}-U_{-}$, where $U_{+}=\max \{U, 0\}$ and $U_{-}=\max \{-U, 0\}$. Since $h$ is convex, $\left(h\left(W+\delta U_{+}\right)-h(W)\right) / \delta$ is pointwise monotonically decreasing as $\delta \searrow 0$, and likewise $(h(W-$ $\left.\left.\delta U_{-}\right)-h(W)\right) / \delta$ is pointwise monotonically increasing. So the interchange of limit and expectation follows from the monotone convergence theorem.

The lemma follows easily from the claim we just proved.

Lemma 5.2. Let $H$ be a graph with $m$ edges, and $0<r \leq 1$. Let $W$ minimize $\operatorname{LT}(H, r)$. Then $W \geq r^{m r^{-m}}$ almost everywhere.

Proof. Let $c=r^{m r^{-m}}$. Suppose on the contrary that $W<c$ on a set of positive measure. Let $\lambda$ be the Lagrange multiplier in Lemma 5.1. From (5.1) we have $t^{\prime}(H, W) \leq m$ everywhere. By considering a positive-measure set of $(x, y)$ such that $W(x, y)<c$, we find

$$
0=h^{\prime}(W(x, y))+\lambda t^{\prime}(H, W)(x, y)<h^{\prime}(c)+m \lambda .
$$

So that

$$
\lambda>\frac{-h^{\prime}(c)}{m}=\frac{\log (1 / c)}{m} .
$$

Therefore, up to a set of measure zero, for every $(x, y)$ with $W(x, y) \geq r^{m}$, we have

$$
t^{\prime}(H, W)(x, y)=\frac{-h^{\prime}(W(x, y))}{\lambda}<\frac{-m h^{\prime}\left(r^{m}\right)}{\log (1 / c)}=\frac{m \log \left(r^{-m}\right)}{\log \left(r^{-m r^{-m}}\right)}=m r^{m} .
$$

On the other hand, for every $(x, y)$ with $W(x, y)<r^{m}$, we have $t^{\prime}(H, W)(x, y) W(x, y)<m r^{m}$. Thus $t^{\prime}(H, W) W<m r^{m}$ almost everywhere. By (5.1) and (5.2), we have

$$
t(H, W)=\frac{1}{m} \mathbb{E}\left[t^{\prime}(H, W) W\right]<r^{m} .
$$

However, any $W$ with $t(H, W)<r^{m}$ cannot minimize $\mathrm{LT}(H, r)$. This gives the desired contradiction.

Lemma 5.3. If $t(H, W) \leq r^{e(H)}$, then $\mathbb{E}[\log W] \leq \log r$.

\footnotetext{
${ }^{9}$ We sketch here an alternate proof of the fact that the minimum is always attained. Let $W_{n}$ be a sequence of graphons with $t\left(H, W_{n}\right) \geq r^{e(H)}$ and $\mathbb{E}\left[h\left(W_{n}\right)\right] \rightarrow \mathrm{LT}(H, r)$. By compactness of the space of graphons 26., there exists a subsequential limit $W$ so that $\delta_{\square}\left(W_{n}, W\right) \rightarrow 0$ along some subsequence. Restrict to this subsequence. We have $t\left(H, W_{n}\right) \rightarrow t(H, W)$, so that $t(H, W) \geq r^{e(H)}$. It remains to show that $\mathbb{E}[h(W)] \leq \lim \mathbb{E}\left[h\left(W_{n}\right)\right]=\mathrm{LT}(H, r)$. We do not lose anything by assuming that $\left\|W_{n}-W\right\|_{\square} \rightarrow 0$. Let $\mathcal{P}_{m}$ denote the partition of the unit interval $[0,1]$ into $m$ equal-length intervals. Let $W_{\mathcal{P}_{m}}$ denote $W$ with its value inside each $I_{i} \times I_{j}$ replaced by its average, for every $I_{i}, I_{j} \in \mathcal{P}_{m}$. Define $\left(W_{n}\right)_{\mathcal{P}_{m}}$ similarly. Then $\left\|W_{n}-W\right\|_{\square} \rightarrow 0$ implies that $\left(W_{n}\right)_{\mathcal{P}_{m}} \rightarrow W_{\mathcal{P}_{m}}$ pointwise a.e. as $n \rightarrow \infty$. By convexity, we have $\lim _{n \rightarrow \infty} \mathbb{E}\left[h\left(W_{n}\right)\right] \geq \liminf _{n \rightarrow \infty} \mathbb{E}\left[h\left(\left(W_{n}\right)_{\mathcal{P}_{m}}\right)\right]=\mathbb{E}\left[h\left(W_{\mathcal{P}_{m}}\right)\right]$. Furthermore, $W_{\mathcal{P}_{m}} \rightarrow W$ pointwise a.e. by the Lebesgue density theorem, so $\lim _{m \rightarrow \infty} \mathbb{E}\left[h\left(W_{\mathcal{P}_{m}}\right)\right]=\mathbb{E}[h(W)]$. It follows that $\mathbb{E}[h(W)] \leq \lim \mathbb{E}\left[h\left(W_{n}\right)\right]=\mathrm{LT}(H, r)$, as desired.
} 


\begin{tabular}{ccccccccccc}
\hline$m$ & 3 & 4 & 5 & 6 & 7 & 8 & 9 & 10 & 20 & 100 \\
\hline$r_{m}$ & 0.686 & 0.735 & 0.770 & 0.795 & 0.815 & 0.831 & 0.844 & 0.855 & 0.911 & 0.973 \\
\hline
\end{tabular}

TABLE 1. Some values of $r_{m}$ from Proposition 5.4 .

Proof. The lemma follows from Jensen's inequality:

$$
\begin{aligned}
m \mathbb{E}[\log W] & =\int_{[0,1]^{V(H)}} \log \left(\prod_{i j \in E(H)} W\left(x_{i}, x_{j}\right)\right) \prod_{i \in V(H)} d x_{i} \\
& \leq \log \left(\int_{[0,1]^{V(H)}} \prod_{i j \in E(H)} W\left(x_{i}, x_{j}\right) \prod_{i \in V(H)} d x_{i}\right)=\log t(H, W) \leq m \log r .
\end{aligned}
$$

Proposition 5.4. Let $H$ be a graph with $m \geq 1$ edges. Let $r=r_{m}$ (see Table 1) be the unique solution in the interval $(0,1)$ to the equation

$$
h\left(r^{m r^{-m}}\right)=h(r)+r^{\prime} h(r)\left(\log \left(r^{m r^{-m}}\right)-\log r\right)
$$

Then $\mathrm{LT}(H, r)$ is uniquely minimized by the constant function $W \equiv r$ for all $r \geq r_{m}$.

Proof. Let $r \geq r_{m}$. Let $W$ be a minimizer for $\operatorname{LT}(H, r)$. By Lemma 5.2 . $W \geq r^{m r^{-m}}$ almost everywhere. Thus it follows by Fact 5.5 below (and it can be checked that $r_{m} \geq 1 / e$ ) that

$$
h(W) \geq h(r)+r \log r(\log W-\log r) \quad \text { a.e. }
$$

Taking expectation of both sides and using $\mathbb{E}[\log W] \leq \log r$ from Lemma 5.3 (note that $\log r \leq 0$ ), we obtain $\mathbb{E}[h(W)] \geq h(r)$, as desired. To see that $W \equiv r$ is unique, suppose $W$ is another minimizer of $\mathbf{L T}(H, r)$. Equality must hold everywhere in the argument. In particular, (5.3) must hold almost everywhere, which easily implies that $W \equiv r$ (for the critical case $r=r_{m}, W$ might also take the value $r^{m r^{-m}}$, but only on a set of measure zero since $\left.\mathbb{E}[h(W)]=h(r)\right)$.

Fact 5.5. If

$$
h(x) \geq h(r)+r h^{\prime}(r)(\log x-\log r)
$$

holds for some $(x, r)=\left(x_{0}, r_{0}\right)$, with $0 \leq x_{0} \leq r_{0} \leq 1$ and $r_{0} \in[1 / e, 1]$, then it holds for all $(x, r) \in\left[x_{0}, 1\right] \times\left[r_{0}, 1\right]$.

Proof. The partial derivative of the RHS of (5.4) with respect to $r$ is $-(1+\log r)(\log r-\log x)$, which is at most zero as long as $x \leq r$ and $r \geq 1 / e$. This shows that if (5.4) holds for some $(x, r)=\left(x_{0}, r_{0}\right)$ then it automatically holds for $(x, r)=\left(x_{0}, r\right)$ for all $r \in\left[r_{0}, 1\right]$.

Let us now fix $r$. Let

$$
f(x):=f_{r}(x):=h(x)-h(r)-r h^{\prime}(r)(\log x-\log r) .
$$

Some examples of $f_{r}$ are plotted in Figure 5. We have

$$
f^{\prime}(x)=\log x-\frac{r \log r}{x} \quad \text { and } \quad f^{\prime \prime}(x)=\frac{x+r \log r}{x^{2}} .
$$

So $f^{\prime \prime}(x)<0$ for $x<-r \log r$ and $f^{\prime \prime}(x)>0$ for $x>-r \log r$. Note also that $f(r)=f^{\prime}(r)=0$, and $-r \log r \leq r$ as long as $r \geq 1 / e$. By analyzing the convexity of $f$ (see Figure 5), we see that $f\left(x_{0}\right) \geq 0$ implies $f(x) \geq 0$ for all $x \in\left[x_{0}, 1\right]$. 

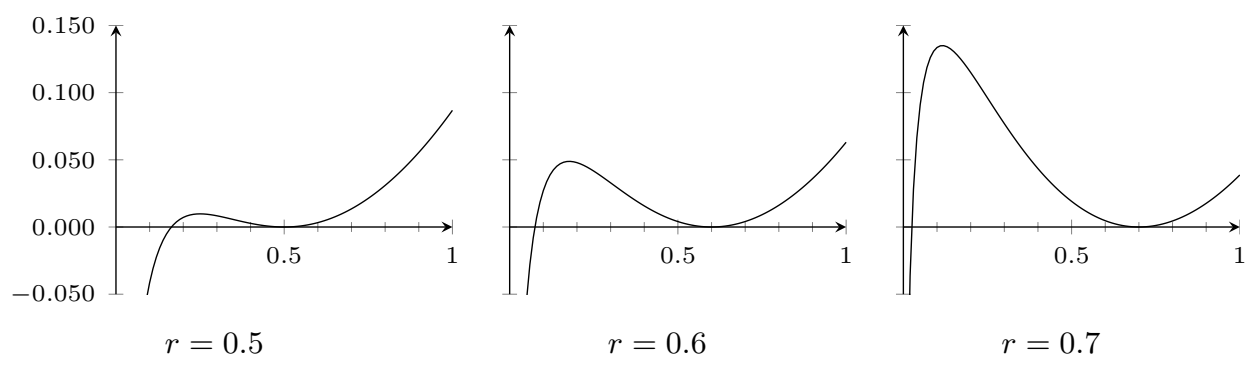

Figure 5. Plot of $f_{r}$ from 5.5 for various values of $r$.

5.2. Symmetry breaking. The proof of the second part of Theorem 2.7 is easy. One can fine tune the bounds as in Section 4.2 though we omit the analysis here.

Proposition 5.6. Let $H$ be a nonbipartite graph. Then $\mathrm{LT}(H, r)<h(r)$ for all $r<0.186$.

Proof. The graphon $W=\mathrm{BIP}_{0,1}$ satisfies $t(H, W)=0$, and $\mathbb{E}[h(W)]=\frac{1}{2} h(0)$, which is strictly less than $h(r)$ for all $r<0.186$.

\section{Open problems}

We conclude with some open problems concerning the variational problem for upper and lower tails.

- Upper tail phase diagram. Determine the upper tail replica symmetry phase diagram for non-regular $H$.

- Lower tail phase diagram. Determine the lower tail replica symmetry phase diagram for $K_{3}$, and more generally for any non-bipartite graph $H$. In particular, determine $r_{H}^{*}$ from Conjecture 2.4. For a bipartite graph $H$, determine whether there is replica symmetry everywhere (Conjecture 2.3).

- Solution in the symmetry breaking phase. Solve the variational problem UT or LT at any non-trivial point where the constant graphon is not a minimizer.

Acknowledgments. I would like to thank Eyal Lubetzky for helpful discussions.

\section{REFERENCES}

[1] D. Aristoff and C. Radin. Emergent structures in large networks. J. Appl. Probab., 50(3):883-888, 2013.

[2] D. Aristoff and L. Zhu. Asymptotic structure and singularities in constrained directed graphs. arXiv:1405.2466.

[3] D. Aristoff and L. Zhu. On the phase transition curve in a directed exponential random graph model. arXiv:1404.6514.

[4] C. Borgs, J. T. Chayes, L. Lovász, V. T. Sós, and K. Vesztergombi. Convergent sequences of dense graphs. I. Subgraph frequencies, metric properties and testing. Adv. Math., 219(6):1801-1851, 2008.

[5] C. Borgs, J. T. Chayes, L. Lovász, V. T. Sós, and K. Vesztergombi. Convergent sequences of dense graphs II. Multiway cuts and statistical physics. Ann. of Math. (2), 176(1):151-219, 2012.

[6] S. Chatterjee. The missing log in large deviations for triangle counts. Random Structures Algorithms, 40(4):437451, 2012.

[7] S. Chatterjee and A. Dembo. Nonlinear large deviations. arXiv:1401.3495.

[8] S. Chatterjee and P. S. Dey. Applications of Stein's method for concentration inequalities. Ann. Probab., 38(6):2443-2485, 2010.

[9] S. Chatterjee and P. Diaconis. Estimating and understanding exponential random graph models. Ann. Statist., to appear.

[10] S. Chatterjee and S. R. S. Varadhan. The large deviation principle for the Erdős-Rényi random graph. European J. Combin., 32(7):1000-1017, 2011.

[11] D. Conlon, J. Fox, and B. Sudakov. An approximate version of Sidorenko's conjecture. Geom. Funct. Anal., 20(6):1354-1366, 2010. 
[12] B. DeMarco and J. Kahn. Upper tails for triangles. Random Structures Algorithms, 40(4):452-459, 2012.

[13] A. W. Goodman. On sets of acquaintances and strangers at any party. Amer. Math. Monthly, 66:778-783, 1959.

[14] H. Hatami. Graph norms and Sidorenko's conjecture. Israel J. Math., 175:125-150, 2010.

[15] S. Janson. Poisson approximation for large deviations. Random Structures Algorithms, 1(2):221-229, 1990.

[16] S. Janson, T. Łuczak, and A. Ruciński. Random graphs. Wiley-Interscience Series in Discrete Mathematics and Optimization. Wiley-Interscience, New York, 2000.

[17] S. Janson and L. Warnke. The lower tail: Poisson approximation revisited. arXiv:1406.1248.

[18] G. Katona. A theorem of finite sets. In Theory of graphs (Proc. Colloq., Tihany, 1966), pages 187-207. Academic Press, New York, 1968.

[19] R. Kenyon, C. Radin, K. Ren, and L. Sadun. Multipodal structure and phase transitions in large constrained graphs. arXiv:1405.0599.

[20] R. Kenyon and M. Yin. On the asymptotics of constrained exponential random graphs. arXiv:1406.3662.

[21] J. Kim, C. Lee, and J. Lee. Two approaches to sidorenko's conjecture. Trans. Amer. Math. Soc. to appear.

[22] J. B. Kruskal. The number of simplices in a complex. In Mathematical optimization techniques, pages $251-278$. Univ. of California Press, Berkeley, Calif., 1963.

[23] J. L. X. Li and B. Szegedy. On the logarithmic calculus and sidorenko's conjecture. Combinatorica. to appear.

[24] L. Lovász. Large networks and graph limits, volume 60 of American Mathematical Society Colloquium Publications. American Mathematical Society, Providence, RI, 2012.

[25] L. Lovász and B. Szegedy. Limits of dense graph sequences. J. Combin. Theory Ser. B, 96(6):933-957, 2006.

[26] L. Lovász and B. Szegedy. Szemerédi's lemma for the analyst. Geom. Funct. Anal., 17(1):252-270, 2007.

[27] E. Lubetzky and Y. Zhao. On the variational problem for upper tails in sparse random graphs. arXiv:1402.6011.

[28] E. Lubetzky and Y. Zhao. On replica symmetry of large deviations in random graphs. Random Structures Algorithms, to appear.

[29] V. Nikiforov. The number of cliques in graphs of given order and size. Trans. Amer. Math. Soc., 363(3):1599-1618, 2011.

[30] C. Radin, K. Ren, and L. Sadun. The asymptotics of large constrained graphs. J. Phys. A, 47(17):175001, 20, 2014.

[31] C. Radin and L. Sadun. Singularities in the entropy of asymptotically large simple graphs. J. Stat. Phys. to appear.

[32] C. Radin and L. Sadun. Phase transitions in a complex network. J. Phys. A, 46(30):305002, $12,2013$.

[33] C. Radin and M. Yin. Phase transitions in exponential random graphs. Ann. Appl. Probab., 23(6):2458-2471, 2013.

[34] A. A. Razborov. On the minimal density of triangles in graphs. Combin. Probab. Comput., 17(4):603-618, 2008.

[35] C. Reiher. The clique density theorem. arXiv:1212.2454.

[36] A. Sidorenko. A correlation inequality for bipartite graphs. Graphs Combin., 9(2):201-204, 1993.

[37] B. Szegedy. Relative entropy and Sidorenko's conjecture. arXiv:1406.6738.

[38] A. Thomason. A disproof of a conjecture of Erdős in Ramsey theory. J. London Math. Soc. (2), 39(2):246-255, 1989.

[39] M. Yin. Large deviations and exact asymptotics for constrained exponential random graphs. arXiv:1412.6001.

[40] M. Yin. Critical phenomena in exponential random graphs. J. Stat. Phys., 153(6):1008-1021, 2013.

[41] M. Yin, A. Rinaldo, and S. Fadnavis. Asymptotic quantization of exponential random graphs.

[42] M. Yin and L. Zhu. Asymptotics for sparse exponential random graph models. arXiv:1411.4722.

[43] M. Yin and L. Zhu. Reciprocity in directed networks. arXiv:1412.2187.

[44] L. Zhu. Asymptotic structure of constrained exponential random graph models. arXiv:1408.1536.

Department of Mathematics, Mit, Cambridge, MA 02139.

E-mail address: yufeiz@mit.edu 